\title{
APTIDÃO FÍSICA RELACIONADA À SAÚDE DE JOVENS CEGOS EM ESCOLAS REGULARES E ESPECIAIS
}

\section{HEALTH PHYSICAL FITNESS OF BLIND ADOLESCENTS IN REGULAR AND SPECIAL SCHOOLS}

Márcia Greguol ${ }^{1}$

Dante de Rose Júnior ${ }^{2}$

Greguol M, Junior DR. Aptidão física relacionada à saúde de jovens cegos em escolas regulares e especiais. Rev Bras Crescimento Desenvolv Hum. 2009; 19(1): 42-53

\section{Resumo:}

A inclusão de alunos com deficiências nas escolas regulares tem atraído a atenção dos educadores. Em algumas escolas, alunos com deficiências são inseridos em turmas regulares, porém sem efetiva participação nas atividades. Ainda como fator agravante, sabe-se que jovens com deficiência em geral apresentam seu lazer extremamente passivo, com poucas oportunidades de vivências motoras. Visando a analisar a situação de alunos com deficiência participando de aulas de educação física, este estudo objetivou a análise de variáveis da aptidão física relacionadas à saúde de adolescentes cegos. Para tanto, 24 adolescentes cegos com idades entre 14 e 16 anos, sendo 12 de escolas regulares estaduais e 12 de uma escola especial privada, tiveram mensuradas algumas variáveis antropométricas, flexibilidade, força muscular e resistência cardiorrespiratória. Ambos os grupos de alunos participavam das aulas de educação física com uma frequência de duas vezes por semana. Foi verificado que, nas variáveis força muscular e resistência cardiorrespiratória, os adolescentes da escola especial apresentaram resultados superiores e melhor evolução do que seus colegas das escolas regulares. Pelos resultados obtidos conclui-se que os adolescentes da escola especial apresentaram melhor nível de aptidão física relacionada à saúde do que seus colegas inseridos em escolas regulares.

Palavras-chave: aptidão física; adolescência; cegueira.

1 Centro de Educação Física e Esporte - Universidade Estadual de Londrina. Departamento de Esporte. e-mail: mgreguol@gmail.com

2 Escola de Artes, Ciências e Humanidades - Universidade de São Paulo. E-mail: danrose@usp.br.

Correspondência para: Dante de Rose Júnior. Escola de Artes, Ciências e Humanidades - Universidade de São Paulo. Rua Arlindo Béttio, n.1000, Ermelino Matarazzo, São Paulo - SP, CEP 03828-000

E-mail: danrose@usp.br 


\begin{abstract}
:
Inclusion of handicap students in regular schools has attracted teacher's attention. In some schools, handicap student are inserted in regular classes, nevertheless without an effective participation in activities. As an aggravate factor, it's known that handicap children generally present an extremely passive leisure, with little motor experiences. In order to verify the handicap students situation in physical education classes, the aim of this study was to analyze health-related physical fitness variables of blind adolescents. Twenty-four blind adolescents, 12 of regular state schools e 12 of one special private school, with ages between 14 and 16 years old, had measured some anthropometric variables, flexibility, muscular strength and cardio-respiratory endurance. Both groups participated in physical education classes twice per week. It was observed that special school adolescents presented better results in muscular strength and cardio-respiratory endurance than the ones in regular school. By the results, it's possible to conclude that special school adolescents presented better levels of health-related physical fitness than others in regular schools.
\end{abstract}

Key word: physical fitness; adolescence; blindness.

\section{INTRODUÇÃO}

O Censo Escolar de 2002 mostra que apenas 5\% das crianças brasileiras estão fora das escolas, entretanto aquelas com deficiências (cerca de seis milhões em idade escolar) não são computadas nesse montante, visto que apenas 400.000 frequentam escolas regulares e 100.000 escolas especiais. ${ }^{1,2}$ Embora existam poucos dados oficiais, sabe-se que grande parte das crianças e adolescentes com deficiências raramente teve acesso ou oportunidade de frequentar uma escola, quer regular, quer especial. Das que conseguem ter acesso à escola, muitas são dispensadas pelos professores das aulas de educação física, com o falso pretexto de "garantir a sua integridade física”. Das que conseguem participar das aulas de educação física, muitas são relegadas para a realização de atividades paralelas, enquanto todos os demais alunos participam de alguma atividade diferente. Esta realidade vai contra as determinações da legislação nacional ${ }^{3,4}$, que já há mais de uma década garante o acesso de todos os alunos às escolas regulares, independente de possuírem algum tipo de deficiência.
Um argumento fortemente utilizado por aqueles que defendem a inclusão de todas as crianças no sistema regular de ensino é que esta prática pode beneficiar a criança com deficiência sob diversos aspectos, como em relação à melhora da sua integração social e ao ganho de autonomia. Entretanto, alguns estudos recentes têm verificado que em muitas situações os objetivos dos alunos com deficiências, no que se refere à inserção efetiva nas atividades e à melhora da aptidão física, não são alcançados nas aulas de educação física escolar. Gorgatti $^{5}$ e Skaggs e Hooper ${ }^{6}$, analisando estudantes cegos em escolas regulares e especiais, verificaram que aqueles das escolas especiais demonstravam melhores índices de aptidão física relacionada à saúde do que seus colegas matriculados em escolas regulares. Além disso, também demonstravam maior satisfação e competência percebida nas aulas e uma melhor compreensão sobre a importância da disciplina de educação física.

Muitos estudos na literatura mostram que pessoas cegas apresentam níveis de força, resistência cardiorrespiratória, velocidade e equilíbrio abaixo da média das videntes da mesma faixa etária. ${ }^{7-14}$ Entretanto, existe tam- 
bém certa unanimidade entre os autores de que uma estimulação adequada e precoce pode amenizar o atraso existente, em grande parte ou até de forma total.

Jovens com deficiências de um modo geral são pouco encorajados pelos pais e professores a praticar atividades físicas e esportivas ${ }^{15,16}$. Embora se saiba que a prática regular de atividades físicas melhora a aptidão física relacionada à saúde, contribuindo assim para o desenvolvimento motor e social, poucas são as oportunidades oferecidas àqueles com deficiência visual para o engajamento em programas que ofereçam o estímulo necessário para o aprimoramento da condição física.

Nos países em desenvolvimento como o Brasil, estima-se que poucas crianças com deficiências tenham acesso à prática de atividades físicas nas escolas regulares, o que dificulta ainda mais que se tornem adultos fisicamente ativos. O estilo de vida sedentário da maioria dos jovens com deficiência reflete diretamente em seu nível de aptidão física e, conseqüentemente, em sua saúde.

Tendo em vista a atualidade do tema, o objetivo deste estudo foi avaliar a evolução de componentes da aptidão física relacionada à saúde de adolescentes cegos em aulas de educação física inclusiva e especial. Ainda, objetivou-se comparar os resultados obtidos pelos adolescentes nos dois sistemas de ensino, a fim de se verificar em qual ambiente (inclusivo ou segregado) os jovens cegos demonstravam melhores resultados.

\section{MÉTODO}

\section{Participantes}

O estudo contou com uma amostra de 24 adolescentes com idades variando de 14 a 16 anos na ocasião do primeiro teste e que apresentavam cegueira de origem congênita. Essa amostra foi dividida em dois grupos: G1, com 12 adolescentes cegos e tendo aulas de educação física em escolas regulares; e G2, com 12 adolescentes cegos e tendo aulas de educação física em uma escola especial. Os grupos (G1 e G2) foram constituídos por adolescentes do sexo masculino, classificados educacionalmente como cegos, ou seja, capazes de ler e escrever apenas pelo código Braille. ${ }^{17}$ Ainda ambos os grupos participavam das aulas de educação física com uma frequência de duas vezes por semana, com cada aula durando 50 minutos. Os sujeitos da amostra participavam ativamente das aulas de educação física em suas escolas e não apresentavam deficiências múltiplas ou comprometimento cognitivo. A restrição no tamanho da amostra deveu-se em grande parte ao fato de que muitas crianças e adolescentes cegos, ainda que matriculados em escolas particulares, eram dispensados das aulas de educação física. Também foram excluídos da amostra adolescentes que participavam de treinamentos especializados para competições de alto rendimento ou que praticavam atividades físicas regulares fora do ambiente escolar.

Os adolescentes cegos avaliados das escolas especiais eram alunos do Instituto de Cegos Padre Chico, na cidade de São Paulo, onde participavam de aulas de educação física. Já os das escolas regulares eram provenientes dos estabelecimentos de ensino públicos da cidade de São Paulo. Para localizar estes adolescentes nas escolas regulares, foi possível contar com levantamentos da Secretaria da Educação e de algumas instituições que têm coletado, nos últimos anos, dados sobre a situação da inclusão nas escolas do Estado de São Paulo.

\section{Procedimentos}

Com relação aos aspectos da aptidão física relacionada à saúde, estes foram acompanhados por meio dos seguintes procedimentos de medição, de acordo com os critérios da bateria Eurofit ${ }^{18}$ : 
- Composição corporal: estatura (cm) em estadiômetro de madeira com precisão de 0,1 centímetro; massa corporal (kg) em balança digital da marca Tanita com precisão de 100 gramas; Índice de Massa Corporal (IMC - kg/ $\mathrm{m}^{2}$ ), calculado dividindo-se o valor da massa em quilogramas pelo quadrado da estatura em metros; e adiposidade, estimada através da soma de sete dobras cutâneas (tricipital, subescapular, suprailíaca, abdominal, peitoral, coxa média e panturrilha medial - em milímetros (mm);

- Resistência muscular: teste de 30 segundos de resistência abdominal, por meio de flexões de tronco (maior número de repetições, em apenas uma tentativa);

- Resistência cardiorrespiratória: teste de nove minutos de corrida - distância em metros (com guia segurando em uma corda elástica; apenas uma tentativa);

- Flexibilidade: teste de sentar e alcançar no banco de Wells - capacidade de flexão de quadril (melhor de três tentativas em $\mathrm{cm}$ ).

Os testes relativos à antropometria e à aptidão física nessa etapa foram aplicados em quatro momentos distintos: no início da pesquisa, após uma semana, após nove meses e após 16 meses. Todas as medidas dos testes foram verificadas por três observadores diferentes ao mesmo tempo, todos profissionais de educação física. A objetividade dos testes foi verificada por não existirem diferenças significativas entre as medições dos três observadores ( $p>0,05)$. Também a reprodutibilidade dos testes foi verificada, visto ser o Coeficiente de Correlação Intraclasses acima de 0,90 para todos os testes comparando-se as medições com uma semana de intervalo. Já a validação dos testes foi feita por conteúdo, solicitando-se a opinião de cinco profissionais de educação física especialistas na área de medidas e avalia- ção e de educação física para pessoas com deficiência. Todos os especialistas consultados foram unânimes em afirmar que os testes empregados eram viáveis, de fácil compreensão e de fato mensuravam as variáveis às quais se propunham para a população pesquisada. Ao final dos períodos de nove e 16 meses, foi avaliada a evolução dos adolescentes relacionada ao tempo e ao tipo de programa de educação física (regular ou especial).

A presente pesquisa foi aprovada pelo Comitê de Ética em Pesquisa da Escola de Educação Física da Universidade de São Paulo (Parecer número 89/04).

\section{Análises estatísticas}

Após ser verificada a normalidade dos dados através do teste de Kolmogorov-Smirnov, os resultados foram comparados em cada uma das três medições e em seguida quanto à sua evolução total. Os testes para as variáveis coletadas no acompanhamento foram avaliados utilizandose análise de variância (ANOVA) para medidas repetidas, para observar a evolução do aluno dentro da escola nos três momentos de testagem e o teste t-Student não-pareado, para a comparação dos resultados entre as escolas regulares e especial. Em ambos os casos o nível de significância adotado foi $\mathrm{p}<0,05$. Para avaliar a evolução dos alunos, foram definidos: Evolução do $2^{\circ}$ período: diferença entre a média do primeiro e do segundo períodos; Evolução do $3^{\circ}$ período: diferença entre a média do segundo e do terceiro períodos; Evolução total: diferença entre a média do primeiro e do terceiro períodos.

Também foram comparados os desempenhos entre as escolas dentro de cada período, definidos como: Diferença 1: média do valor nas escolas regulares - média do valor na escola especial no tempo 1; Diferença 2: média do valor nas escolas regulares - média do valor na escola especial no tempo 2; Diferença 3: média do valor nas escolas regulares - média do valor na escola especial no tempo 3. 


\section{RESULTADOS}

\section{Evolução da composição corporal}

As variáveis antropométricas verificadas foram: massa corporal (kg), estatura (cm), Índice de Massa Corporal (IMC) e soma de sete dobras cutâneas em milímetros (tricipital, peitoral, subescapular, suprailíaca, abdominal, da coxa média e da panturrilha medial). As tabelas 1, 2, 3 e 4 mostram, respectivamente, os resultados médios das variáveis massa, estatura, IMC e soma de dobras cutâneas. Não houve diferenças significativas entre os grupos em nenhuma das variáveis analisadas.

Tabela 1: Valores médios e desvio padrão da massa corporal dos adolescentes nas três medições.

\begin{tabular}{cccc}
\hline & \multicolumn{3}{c}{ Escola } \\
Medição & Regular & Especial & Geral \\
\hline 1 & $62,37(10,86)$ & $59,93(9,48)$ & $61,15(10,14)$ \\
2 & $64,21(9,52)$ & $62,75(9,06)$ & $63,48(9,20)$ \\
3 & $65,17(8,81)$ & $63,45(8,25)$ & $64,31(8,47)$ \\
\hline
\end{tabular}

Tabela 2: Valores médios e desvio padrão da estatura dos adolescentes nas três medições.

\begin{tabular}{cccc}
\hline & \multicolumn{3}{c}{ Escola } \\
Medição & Regular & Especial & Geral \\
\hline 1 & $168,26(5,46)$ & $169,58(6,04)$ & $168,92(5,73)$ \\
2 & $171,84(5,20)$ & $173,16(5,74)$ & $172,90(5,45)$ \\
3 & $174,85(4,86)$ & $175,00(5,63)$ & $174,93(5,22)$ \\
\hline
\end{tabular}

Tabela 3: Valores médios e desvio padrão do IMC dos adolescentes nas três medições.

\begin{tabular}{cccc}
\hline & \multicolumn{3}{c}{ Escola } \\
Medição & Regular & Especial & Geral \\
\hline 1 & $21,94(3,00)$ & $20,85(3,20)$ & $21,39(3,11)$ \\
2 & $21,84(2,54)$ & $21,10(3,07)$ & $21,47(2,81)$ \\
3 & $21,93(2,35)$ & $21,13(2,80)$ & $21,53(2,59)$ \\
\hline
\end{tabular}

Tabela 4: Valores médios e desvio padrão da soma de sete dobras cutâneas dos adolescentes nas três medições.

\begin{tabular}{cccc}
\hline & \multicolumn{3}{c}{ Escola } \\
Medição & Regular & Especial & Geral \\
\hline 1 & $102,5(17,29)$ & $92,9(13,72)$ & $97,7(15,17)$ \\
2 & $99,6(10,03)$ & $92,0(16,38)$ & $95,8(17,92)$ \\
3 & $99,5(14,89)$ & $91,8(14,60)$ & $95,6(14,47)$ \\
\hline
\end{tabular}

\section{Evolução das características da aptidão física}

A idéia nessa etapa foi verificar em qual tipo de escola (regular ou especial) o adolescente cego alcançava melhores índices de aptidão física relacionada à saúde e em qual delas demonstrava uma melhor evolução nessas variáveis. Cabe ressaltar que os adolescentes avaliados não participavam de programas regulares de atividades físicas, exceto das aulas de educação física nas escolas.

As tabelas 5, 6 e 7 mostram, respectivamente, os resultados médios da variável flexibilidade, a sua evolução durante as três medições e as diferenças dos resultados entre as escolas regulares e especial.

Tabela 5: Valores médios da flexibilidade e sua evolução para os dois grupos.

\begin{tabular}{cccc}
\hline & \multicolumn{3}{c}{ Escola } \\
Medição & Regular & Especial & Geral \\
\hline 1 & $14,55(5,49)$ & $16,10(7,30)$ & $15,33(6,43)$ \\
2 & $15,65(5,82)$ & $16,40(6,81)$ & $16,02(6,07)$ \\
3 & $16,10(5,28)$ & $17,20(6,95)$ & $16,65(6,12)$ \\
\hline
\end{tabular}

Tabela 6: Evolução da variável flexibilidade nas três medições.

\begin{tabular}{lcccc}
\hline & & $\begin{array}{c}\text { Evolução } \\
\text { Período }\end{array}$ & $\begin{array}{c}\text { Evolução } \\
\text { Período }\end{array}$ & $\begin{array}{c}\text { Evolução } \\
\text { Total }\end{array}$ \\
\hline \multirow{2}{*}{ Regular } & Diferença & 1,10 & 0,45 & 1,55 \\
& $\mathrm{P}$ & $0,037^{*}$ & 0,103 & $0,002^{*}$ \\
\multirow{2}{*}{ Especial } & Diferença & 0,31 & 0,79 & 1,10 \\
& $\mathrm{P}$ & 0,304 & $0,029 *$ & $0,009 *$ \\
\hline
\end{tabular}

* diferença significante $(\mathrm{p}<0,05)$ 
Tabela 7: Diferenças na variável flexibilidade entre as escolas regulares e especial.

\begin{tabular}{cccc}
\hline \multicolumn{3}{c}{ Regular x Especial } \\
\hline & Medição 1 & Medição 2 & Medição 3 \\
Diferença & $-1,55$ & $-0,76$ & $-1,10$ \\
P & 0,433 & 0,708 & 0,561 \\
\hline
\end{tabular}

Os valores médios da variável flexibilidade foram estimados pela distância que o adolescente atingia no teste de sentar e alcançar no banco de Wells. Os resultados apresentados dessa variável mostram que não houve diferenças significativas entre o desempenho de adolescentes da escola especial e os das escolas regulares. Quanto à evolução através das três medições, os alunos das escolas regulares mostravam uma melhor evolução entre a primeira e a segunda medições e no período de tempo total. Já os da escola especial mostraram uma evolução significativa entre a segunda e a terceira medições e no período de tempo total. A evolução total na capacidade flexibilidade foi semelhante nos dois tipos de escola, entretanto houve uma tendência de melhora mais expressiva para os adolescentes das escolas regulares (1,5 cm em média de evolução total ou $10,6 \%$, contra $1,10 \mathrm{~cm}$ ou $6,8 \%$ para os da escola especial).

As tabelas 8, 9 e 10 mostram, respectivamente, os resultados médios da variável resistência abdominal, a sua evolução durante as três medições e as diferenças dos resultados entre as escolas regulares e especial.

Tabela 8: Valores médios da resistência abdominal e sua evolução para os dois grupos.

\begin{tabular}{cccc}
\hline & \multicolumn{3}{c}{ Escola } \\
Medição & Regular & Especial & Geral \\
\hline 1 & $9,90(2,92)$ & $15,05(4,17)$ & $12,48(4,41)$ \\
2 & $11,75(2,61)$ & $16,85(2,89)$ & $14,30(3,75)$ \\
3 & $12,40(2,33)$ & $19,00(3,54)$ & $15,70(4,46)$ \\
\hline
\end{tabular}

Tabela 9: Evolução da variável resistência abdominal nas três medições.

\begin{tabular}{lcccc}
\hline & & $\begin{array}{c}\text { Evolução } \\
\text { Período }\end{array}$ & $\begin{array}{c}\text { Evolução } \\
\text { Período }\end{array}$ & $\begin{array}{c}\text { Evolução } \\
\text { Total }\end{array}$ \\
\hline \multirow{2}{*}{ Regular } & Diferença & 1,85 & 0,65 & 2,50 \\
& $\mathrm{P}$ & $0,001^{*}$ & $0,017^{*}$ & $0,000^{*}$ \\
\multirow{2}{*}{ Especial } & Diferença & 1,80 & 2,15 & 3,95 \\
& $\mathrm{P}$ & $0,001^{*}$ & $0,000^{*}$ & $0,000^{*}$ \\
\hline
\end{tabular}

* diferença significante $(\mathrm{p}<0,05)$

Tabela 10: Diferenças na variável resistência abdominal entre as escolas regulares e especial.

\begin{tabular}{cccc}
\hline & \multicolumn{3}{c}{ Regular x Especial } \\
& Medição 1 & Medição 2 & Medição 3 \\
\hline Diferença & $-5,15$ & $-5,10$ & $-6,60$ \\
P & $0,000^{*}$ & $0,000^{*}$ & $0,000^{*}$ \\
\hline
\end{tabular}

* diferença significante $(\mathrm{p}<0,05)$

Os valores médios da variável resistência abdominal foram estimados pelo número de exercícios abdominais que os adolescentes conseguiam executar em um intervalo de trinta segundos. Somente foram considerados válidos os movimentos nos quais os adolescentes encostavam os cotovelos nas coxas. Conforme os resultados, os adolescentes da escola especial apresentaram valores significativamente maiores de movimentos do que os das escolas regulares, indicando que sua resistência abdominal foi superior nas três medições. Quanto à evolução, tanto os adolescentes das escolas regulares como os da especial mostraram uma melhora geral e ao longo das três medições na resistência abdominal. Entretanto, aqueles da escola especial mostraram uma leve tendência de melhora acima da obtida por seus colegas das escolas regulares (3,95 movimentos ou $26,2 \%$ contra 2,5 movimentos ou 25,2\% de melhora, respectivamente).

As tabelas 11, 12 e 13 mostram, respectivamente, os resultados médios da variável resistência cardiorrespiratória, a sua 
Tabela 11: Valores médios da corrida de nove minutos e sua evolução para os dois grupos.

\begin{tabular}{cccc}
\hline & \multicolumn{4}{c}{ Escola } \\
Medição & Regular & Especial & Geral \\
\hline 1 & $945,00(158,79)$ & $1263,00(169,49)$ & $1104,00(228,49)$ \\
2 & $1021,00(173,69)$ & $1342,50(156,81)$ & $1181,75(230,61)$ \\
3 & $1030,00(149,17)$ & $1408,00(132,25)$ & $1219,00(236,64)$ \\
\hline
\end{tabular}

Tabela 12: Evolução da variável resistência em nove minutos nas três medições.

\begin{tabular}{lcccc}
\hline & & $\begin{array}{c}\text { Evolução } \\
\text { Período }\end{array}$ & $\begin{array}{c}\text { Evolução } \\
\text { Período }\end{array}$ & $\begin{array}{c}\text { Evolução } \\
\text { Total }\end{array}$ \\
\hline \multirow{2}{*}{ Regular } & Diferença & 76,00 & 9,00 & 85,00 \\
& $\mathrm{P}$ & $0,000^{*}$ & 0,236 & $0,000^{*}$ \\
\multirow{2}{*}{ Especial } & Diferença & 79,50 & 65,50 & 145,00 \\
& $\mathrm{P}$ & $0,000^{*}$ & $0,000^{*}$ & $0,000^{*}$ \\
\hline
\end{tabular}

* diferença significante $(\mathrm{p}<0,05)$

Tabela 13: Diferenças na variável resistência em nove minutos entre as escolas regulares e especial.

\begin{tabular}{cccc}
\hline & \multicolumn{3}{c}{ Regular x Especial } \\
& Medição 1 & Medição 2 & Medição 3 \\
\hline Diferença & $-318,00$ & $-321,50$ & $-378,00$ \\
P & $0,000 *$ & $0,000 *$ & $0,000^{*}$ \\
\hline
\end{tabular}

* diferença significante $(\mathrm{p}<0,05)$

evolução durante as três medições e as diferenças dos resultados entre as escolas regulares e especial.

Os valores médios de resistência cardiorrespiratória foram estimados pela distância em metros que os adolescentes conseguiam percorrer, correndo ou andando, durante um intervalo de tempo de nove minutos. Em todas as medições realizadas, os alunos da escola especial apresentaram valores de resistência geral superiores aos das escolas regulares, conseguindo percorrer distâncias maiores durante os nove minutos de teste. Quanto à evolução, os alunos dos dois tipos de escola me- lhoraram de forma significativa em todas as medições e no geral, com exceção do grupo das escolas regulares entre a segunda e a terceira medições. É importante registrar que a evolução dos alunos da escola especial foi superior à dos das escolas regulares no que se refere à resistência cardiorrespiratória (145m ou $11,5 \%$ contra $85 \mathrm{~m}$ ou $8,9 \%$ de melhora, respectivamente).

\section{DISCUSSÃO}

Vários autores apontam para a necessidade da criação de testes e padrões específicos a fim de que o processo de avaliação seja de fato benéfico e ofereça subsídios relevantes ao professor de educação física. Alguns testes para jovens com deficiências podem expô-los a uma situação frustrante, além de não mensurar de fato suas reais possibilidades de movimento. ${ }^{19-21} \mathrm{~A}$ intenção com o protocolo de avaliação sugerido foi mensurar componentes da aptidão física que estivessem correlacionados a parâmetros positivos de saúde. Block et al ${ }^{22}$ e Maragon ${ }^{23}$, discutindo sobre avaliação em educação física, afirmam que é necessário o professor verificar como crianças e jovens desenvolvem os componentes da aptidão física relacionados à saúde e que os dados obtidos nessas mensurações devem ser utilizados para modificar a direção do programa de educação física.

Para Winnick e Short ${ }^{24}$, jovens com deficiências visuais deveriam possuir, no mínimo, níveis de consumo de oxigênio e composição corporal consistentes com a saúde positiva, flexibilidade para a saúde funcional (especialmente alinhamento pélvico e postura adequados e funcionalidade da coluna lombar) e níveis de força e resistência abdominal adequados para uma vida independente e participação em atividades físicas. 
A análise das variáveis da aptidão física relacionada à saúde é de grande importância para a população em geral, especialmente como forma de se predizer o risco aumentado de doenças hipocinéticas. Para indivíduos com deficiência essa preocupação torna-se mais evidente, uma vez que estes em geral apresentam estilos de vida com forte tendência ao sedentarismo. Para pessoas com deficiência visual, quanto melhores os níveis da aptidão física relacionada à saúde, também provavelmente maior será a autonomia para a realização das atividades de vida diária e, em conseqüência, maior será sua independência e capacidade de inserção social. ${ }^{25}$

As variáveis da aptidão física pesquisadas foram escolhidas levando-se em conta sua facilidade de medição fidedigna em testes de campo e sua relação com parâmetros de saúde. A importância das aulas de educação física para estes jovens é fundamental, uma vez que a maioria deles não tem acesso a outras opções de atividades físicas fora do ambiente escolar. Crianças e adolescentes sem deficiência em geral reúnem-se informalmente com os amigos para brincar e praticar esportes ou então freqüentam clubes e academias com mais facilidade, tendo oportunidade de experimentar uma gama variada de vivências motoras. Já aqueles que são cegos, em muitas situações, são desencorajados pelos próprios pais, que não os estimulam a sair de suas casas e a praticar outras atividades que não aquelas propostas no ambiente escolar. Dessa maneira, para esse segmento da população, as atividades desenvolvidas nas aulas de educação física escolar representam uma parte significativa de suas vivências motoras.

Winnick e Short ${ }^{24}$ destacam que, para jovens com deficiências visuais, os padrões de aptidão física utilizados devem ser os mesmos da população sem deficiência, com exceção dos testes que envolvam corrida ou caminhada. Dessa maneira, buscou-se comparar em cada variável os resultados gerais obtidos aos parâmetros considerados normais pela literatura e compatíveis com a saúde positiva.

Foi verificado que, no que se refere às variáveis antropométricas, não houve diferenças significativas entre as escolas regulares e especial em nenhuma das três medições ( $\mathrm{p}>0,05)$. Nas variáveis massa corporal e estatura, tanto os alunos da escola especial como aqueles de escolas regulares mostraram uma evolução significativa em todas as medições, fato esse totalmente esperado, visto que os adolescentes encontram-se em fase de crescimento. Já os valores de Índice de Massa Corporal (IMC) evoluíram de forma significativa apenas nas medições realizadas com os adolescentes da escola especial, mostrando que, para estes, o valor da massa elevou-se de forma mais expressiva do que o valor da estatura.

Quanto à soma das sete dobras cutâneas, além de não ter sido observada diferença significativa entre as escolas nas diferentes medições, a evolução também não foi expressiva. Em outras palavras, a quantidade de gordura periférica medida em todos os adolescentes não aumentou de maneira significativa durante as três medições, mantendo-se com uma tendência à estabilização. Pode-se supor, dessa forma, que o significativo aumento do IMC entre os adolescentes da escola especial deveu-se ao aumento da massa muscular, visto que a quantidade de gordura não demonstrou elevação expressiva. Estes dados vão ao encontro dos verificados por Brito et al ${ }^{26}$, que observaram em suas pesquisas com adolescentes do sexo masculino que a quantidade total de gordura mantinha-se estável durante a adolescência, diferente do índice de muscularidade, que aumentava de forma significativa. Para os dois grupos pesquisados, os resultados obtidos no IMC nas três medições foram compatíveis com a saúde positiva, concentrando-se entre os percentis 25 e 75 de acordo com as referências do Centers for Disease Control (CDC). ${ }^{27}$ 
Os resultados dos testes de aptidão física realizados demonstraram que, embora os adolescentes cegos da escola especial mostrassem, em geral, aptidão física superior à de seus colegas das escolas regulares em todas as testagens, ambos os grupos apresentaram melhora significativa em praticamente todas as variáveis. Cabe ressaltar que nenhum sujeito da amostra possuía outra vivência motora regular que não as oferecidas nas aulas de educação física na escola e que todos deviam participar das aulas. Entretanto, a melhora dos alunos nos testes de aptidão física pode ter sido devida também aos fatores da experiência e da maturação. Na primeira medição, especialmente os adolescentes das escolas regulares demonstraram-se muito inseguros e receosos em realizar os testes, sobretudo os que envolviam corrida e saltos. Já na última medição, mostraram-se mais seguros e já conheciam bem os procedimentos do teste. Ainda que tenham sido oferecidas chances para treinar os movimentos em todas as medições, certamente a repetição do teste proporcionou uma experiência para os adolescentes. Também cabe ressaltar que os adolescentes, com o avançar da idade, atingiram níveis mais elevados de maturação sexual e, conseqüentemente, de desempenho nos testes.

Ainda que os fatores experiência e maturação possam ter influenciado, a melhora significativa nos resultados dos testes não ocorreu na mesma magnitude para os adolescentes dos diferentes tipos de escolas. Em valores relativos, os resultados de resistência abdominal e resistência geral aeróbia melhoraram de forma mais significativa para os alunos da escola especial ao longo dos 16 meses de avaliação. Apenas no teste de flexibilidade a melhora foi mais significativa para o grupo das escolas regulares.

Os resultados deste estudo mostram a mesma tendência daqueles obtidos por Skaggs e Hooper ${ }^{6}$ que, ao analisarem a aptidão física de jovens com deficiência visual, também ve- rificaram que aqueles das escolas especiais apresentavam melhores resultados do que seus colegas das escolas regulares. Os autores atribuíram seus achados à possibilidade de que as escolas especiais teriam seus programas mais devidamente ajustados às necessidades dos jovens com deficiência visual.

Com relação ao teste de resistência abdominal, Winnick e Short ${ }^{24}$ colocam que jovens de 14 a 16 aos com deficiência visual deveriam atingir o mesmo padrão da população sem deficiência com a mesma faixa etária, neste caso um valor mínimo de 14 repetições. Como se verifica pelos resultados dos adolescentes cegos nesta pesquisa, apenas aqueles da escola especial ficaram acima desse valor mínimo.

Uma das variáveis que demonstrou maiores discrepâncias com relação aos padrões considerados ideais foi a flexibilidade. Houwen et al. ${ }^{28}$, analisando a aptidão física relacionada à saúde de crianças de ambos os sexos com deficiência visual com idades entre 6 e 12 anos, verificaram que estas apresentaram resultados médios para o teste de sentar e alcançar de $27,5 \mathrm{~cm}$, muito superiores aos verificados entre os adolescentes. De acordo com Winnick e Short ${ }^{24}$, a flexibilidade de jovens cegos ou com visão subnormal deveria ser a mesma de jovens videntes. Segundo os autores, jovens com idades de 14 a 16 anos deveriam atingir pelo menos 20 centímetros no teste de sentar e alcançar. Como se verifica pelos resultados, os adolescentes analisados neste estudo ficaram um pouco abaixo deste padrão, sendo que o grupo que mais se aproximou foi o dos alunos da escola especial.

Quando os resultados obtidos neste trabalho foram comparados aos dos adolescentes da mesma faixa etária com visão normal, a maior discrepância ocorreu para as variáveis que envolviam corrida. Observou-se que o padrão de movimento de todos os adolescentes 
cegos era semelhante e precário durante seus deslocamentos. Um jovem cego, por não poder ver o possível perigo, normalmente costuma usar uma base de apoio ampla para evitar quedas ao tropeçar em obstáculos. ${ }^{29} \mathrm{~A}$ base de apoio ampla, também demonstrada durante a corrida, não permite um movimento rápido e pode gerar tensões articulares. Em todos os testes de corrida nas escolas regulares e em alguns da escola especial observou-se que os adolescentes corriam com as pernas afastadas, os pés arrastando no chão e os braços estáticos à frente. Outro fato que pode prejudicar o desempenho na corrida é o fato de um dos braços ficar com os movimentos inibidos pela corda que serve como guia. Apenas alguns alunos da escola especial corriam com um padrão de movimento mais próximo da normalidade. Böhme e Freitas ${ }^{30}$, avaliando jovens em escolas de Viçosa (MG), verificaram que o percentil cinqüenta no teste de corrida de nove minutos para adolescentes de 14 e 16 anos foi, respectivamente, 1639 metros e 1826 metros. Notase pelos resultados dos adolescentes cegos neste estudo que todos ficariam abaixo do percentil 50, quando comparados a adolescentes sem deficiência visual.

Juodzbaliene e Muckus ${ }^{31}$ também avaliaram o desenvolvimento do controle motor de jovens com e sem deficiência visual. Verificaram que este parecia ser dependente do nível de visão e que aqueles totalmente cegos apresentaram os piores resultados para as variáveis tempo de reação e equilíbrio. Entretanto, os autores afirmam que os prejuízos provenientes da falta de visão podem ser em grande parte compensados pela intensificação das funções proprioceptiva, vestibular e auditiva.

A prática regular e bem orientada de exercício físico pode ser vista como uma contribuição importante para a saúde. A grande tarefa da educação física escolar deve ser a de habilitar os alunos a praticar o exercício físico e o esporte e a compreender os determinantes fisiológicos, biomecânicos, socioeconômicos e culturais dessa prática. Somente assim ela estará contribuindo para a ampliação do entendimento do binômio exercício - saúde, para a construção de estilos de vida ativa e para uma sociedade mais justa e igualitária.

Para muitos indivíduos cegos, as aulas de educação física são uma das poucas oportunidades para vivenciarem experiências motoras variadas, o que pode ser fundamental para garantir seu melhor desenvolvimento motor. Infelizmente, pelo que se observa na prática, a educação física parece ainda estar longe de incluir de fato todos os alunos. Justamente ela, que de todas as disciplinas deveria ser uma das mais receptivas à diferença, por admitir inúmeras respostas como sendo corretas. Diferente das equações matemáticas, para as quais existe apenas uma resposta certa, no universo da educação física várias são as possibilidades para se atingir um determinado fim.

\section{CONSIDERAÇÕES FINAIS}

Comparando longitudinalmente, durante 16 meses, 24 adolescentes cegos de escolas regulares e de uma escola especial para cegos, conclui-se que, com relação às variáveis antropométricas, a evolução ocorreu de forma semelhante. Com exceção do Índice de Massa Corporal, que mostrou diferença apenas para os adolescentes da escola especial, as comparações mostraram que todos os sujeitos pesquisados tinham praticamente o mesmo porte físico nos três momentos de avaliação.

Quanto aos componentes da aptidão física, ainda na comparação longitudinal, conclui-se que, em todas as variáveis analisadas, com exceção da flexibilidade, os adolescentes da escola especial mostraram resultados superiores aos dos alunos das escolas regulares. Entretanto, também se pôde verificar que os alunos das escolas regulares também me- 
lhoraram de forma significativa seu desempenho nos testes de aptidão física ao longo do tempo.

Apesar dos resultados, não se nega aqui os ganhos que podem advir de uma inclusão com qualidade. Apenas questiona-se como o processo

\section{REFERÊNCIAS}

1. Clemente CA. Vencendo barreiras histórias de superação e inclusão da pessoa portadora de deficiência. Osasco: Espaço da Cidadania; 2002.

2. Mantoan ME. A hora e a vez da educação inclusiva. Educação e família Deficiências: a diversidade faz parte da vida 2003;1:42-45.

3. Brasil. Constituição da República Federativa do Brasil. Brasília: Imprensa Oficial; 1988.

4. Brasil. Lei de diretrizes e bases da educação. Brasília: Imprensa Oficial; 1996.

5. Gorgatti, MG. Educação física escolar e inclusão: uma análise a partir do desenvolvimento motor e social de adolescentes com deficiência visual e das atitudes dos professores. [Tese de Doutorado - Programa de Pós-graduação em Educação Física e Esporte]. São Paulo (SP): Universidade de São Paulo; 2005.

6. Skaggs S, Hooper C. Individuals with visual impairments: a review of psychomotor behavior. Adapt Phys Activ Q 1996;13:16-26.

7. Hopkins WG, Gaeta H, Thomas AC, McHill P. Physical fitness of blind and sighted children. Eur J Appl Physiol 1987;56(1):69-73.

8. Jankowski LW, Evans JK. The exercise capacity of blind children. J Vis Impair Blind 1981;248-252. tem ocorrido nos últimos anos no Brasil. A situação atual parece mostrar um quadro de despreparo, não apenas no que se refere ao conhecimento, como também no que diz respeito ao currículo da escola, à sua estrutura arquitetônica, aos materiais disponíveis e ao apoio multidisciplinar.

9. Korach O, Tennenbaum A, Schnitzer R, Ornoy A. Early motor development of blind children. J Pediatr Child Health 2000;36(3):226-229.

10. Lopes MCB, Kitadai SPS, Okai LA. Avaliação e tratamento fisioterapêutico das alterações motoras presentes em crianças deficientes visuais. Rev Bras Oftalmol 2004;63(3):155-161.

11. Natale J, Lee M, Ward G, Shephard RJ. Loss of physical conditions in sightless adolescents during a summer vacation. Adapt Phys Activ Q 1985;2:144-150.

12. Sundberg S. Maximal oxygen uptake in relation to age in blind and normal boys and girls. Acta Paediatr Scand 1982;71(4):603-608.

13. Warren DH. Blindness and children: an individual differences approach. Cambridge: Cambridge University; 1994.

14. Griffin-Shirley N, Nes SL. Self-Esteem and Empathy in Sighted and Visually Impaired Preadolescents. J Vis Impair Blind 2005;99:276-285.

15. Robinson BL, Lieberman LJ. Effects of visual impairment, gender and age on selfdetermination. J Vis Impair Blind 2004;98(6):1-32.

16. Shapiro DR, Moffetti A, Lieberman L, Dummer GM. Perceived competence of children with visual impairments. J Vis Impair Blind 2005;99(1):28-54.

17. Bolt D. Blindness and the problems of terminology. J Vis Impair Blind 2002;97(9):519-520. 
18. Eurofit. Handbook for the Eurofit tests and physical fitness. Comittee for Development of Sport. Rome. 1988.

19. Mello MT. Paraolimpíadas: Sidney 2002. São Paulo: Atheneu; 2002.

20. Tan SK, Parker HE, LARKIN D. Concurrent validity of motor tests used to identify children with motor impairment. Adapt Phys Activ Q 2001;18:168-182.

21. Wright H, Sugden D. Physical education for all. London: David Fulton; 1999.

22. Block ME, Lieberman LJ, Kuntz FC. Authentic assessment in adapted physical education. JOPERD 1998;69(3):48-54.

23. Maragon C. Como avaliar na educação física. Rev Nova Escola 2003;maio:50-51.

24. Winnick JP, Short FX. Testes de aptidão física para jovens com necessidades especiais. São Paulo: Manole; 2001.

25. Lieberman LJ. Fitness para personas com discapacidad visual o surdoceguera. Entre dos mundos - Rev Traducción Discapacidad Visual 2003;21:13-22.
26. Brito CFA, Andrade DR, Araújo TL, Matsudo VKR. Estabilidade da aptidão física entre a infância e adolescência. Rev Bras Ativ Fís \& Saúde 1999;4(1):5-12.

27. CDC - Centers for Disease Control and Prevention. National Center for Health Statistics. Advance Data, n.314, Dec. 4, 2000. Disponível em: http://www.cdc.gov/ growthcharts. Acesso em: março 2008.

28. Houwen S, Visscher C, Hartman E, Lemmink KAPM. Test-retest reliability of Eurofit Physical Fitness items for children with visual impairment. Pediatr Exerc Sci 2006;18:300-313.

29. Martín MB, Bueno ST. Deficiência visual: aspectos psicoevolutivos e educativos. São Paulo: Santos; 2003.

30. Böhme MTS, Freitas MC. Aptidão física - avaliação de aspectos relacionados com a saúde. Viçosa: Universidade Federal de Viçosa; 1989.

31. Juodzbaliene V, Muckus K. The influence of the degree of visual impairment on psychomotor reaction and equilibrium maintenance of adolescents. Medicina 2006;42(1):49-56.

Recebido em: 10/10/2008

Modificado em: 15/11/2008

Aceito em: 10/01/2009 